\title{
Borel-Summability of the High Temperature Expansion for Classical Continuous Systems
}

\author{
Wolfgang Wagner \\ Institut für Theoretische Physik der Universität Zürich, Schönberggasse 9, CH-8001 Zürich, \\ Switzerland
}

\begin{abstract}
It is shown that for classical gases with stable, bounded and absolutely integrable pair interactions, the Taylor expansions in $\beta$ of the correlation functions and the pressure are Borel-summable at $\beta=0$.
\end{abstract}

\section{Introduction}

The question of analyticity in $\beta$ for classical continuous systems was considered some years ago by Lebowitz and Penrose [1]. Among other results they showed that for hard core potentials pressure and correlation functions are analytic at $\beta=0$. In this paper we treat the case of bounded potentials, where analyticity is not to be expected, as the expansion is around the ideal gas and the negative of a stable potential is unstable, which causes divergence of the pressure for negative $\beta$ in the finite volume.

\section{Infinite Volume Correlation Functions}

We assume the interaction potential $\phi$ to satisfy stability,

$$
\sum_{\substack{i, j=1 \\ i<j}}^{m} \Phi\left(x_{i}-x_{j}\right) \geqq-m B \quad \text { for some constant } B
$$

and

$$
\begin{gathered}
\|\Phi\|_{\infty}<\infty, \\
\|\Phi\|_{1}<\infty .
\end{gathered}
$$

Eqs. (2) and (3) imply regularity ([2], ch. 4.1):

$$
\int\left|e^{-\beta \Phi(x)}-1\right| d x=C(\beta)<\infty \text { for } \beta \in \mathbb{C} .
$$

We shall use the representation of the correlation functions given by Ruelle ([2], ch. 4.2.):

On the Banach-spaces $E_{\xi}, \xi>0$ of sequences of complex functions $\varphi=$ 
$\left(\varphi(x)_{n}\right)_{n \geqq 1}$ with the norm

we define the operator $\mathbf{K}_{\beta}$ by

$$
\|\varphi\|_{\xi}=\sup _{n \geqq 1}\left(\xi^{-n} \underset{(x)_{n} \in \mathbb{R}^{v n}}{\operatorname{ess} \sup }\left|\varphi(x)_{n}\right|\right),
$$

$$
\begin{aligned}
\left(\mathbf{K}_{\beta} \varphi\right)\left(x_{1}\right)=\sum_{n=1}^{\infty} \frac{1}{n !} \int d(y)_{n} K_{\beta}\left(x_{1},(y)_{n}\right) \varphi(y)_{n} \\
\left(\mathbf{K}_{\beta} \varphi\right)(x)_{m}=\exp \left[-\beta W^{i}(x)_{m}\right] \\
\cdot\left[\varphi(x)_{m-1}^{\prime}+\sum_{n=1}^{\infty} \frac{1}{n !} \int d(y)_{n} K_{\beta}\left(x_{i},(y)_{n}\right) \varphi\left((x)_{m-1}^{\prime},(y)_{n}\right)\right],
\end{aligned}
$$

where $(x)_{m-1}^{\prime}=\left(x_{1}, \ldots, \hat{x}_{i}, \ldots, x_{m}\right)$, the kernel is given by

$$
K_{\beta}\left(x_{i},(y)_{n}\right)=\prod_{j=1}^{n}\left(\exp \left[-\beta \Phi\left(x_{i}-y_{j}\right)\right]-1\right)
$$

and

$$
W^{i}(x)_{m}=\sum_{\substack{j=1 \\ j \neq i}}^{m} \Phi\left(x_{i}-x_{j}\right) .
$$

The index $i$ in (7) depends on $\left(x_{1}, \ldots, x_{m}\right)$ and is chosen so as to ensure

$$
W^{i}(x)_{m} \geqq-2 B,
$$

which is always possible by (1).

For a linear mapping $\mathbf{A}: E_{\eta} \rightarrow E_{\xi}$ we define

$$
\|\mathbf{A}\|_{\eta}^{\xi}=\sup _{\|\varphi\|_{\eta}=1}\|\mathbf{A} \varphi\|_{\xi} .
$$

If $\operatorname{Re} \beta \geqq 0, \mathbf{K}_{\beta}$ is a bounded operator on $E_{\xi}$ and

For

$$
\left\|\mathbf{K}_{\beta}\right\|_{\xi}^{\xi} \leqq e^{2 B \operatorname{Re} \beta} \xi^{-1} \exp [\xi C(\beta)] .
$$

$$
|z|<e^{-2 B \operatorname{Re} \beta} \xi \exp [-\xi C(\beta)]
$$

the sequence $\rho=\left(\rho(x)_{n}\right)_{n \geqq 1}$ of the infinite volume correlation functions belongs to $E_{\xi}$ and can be written as

$$
\rho(\beta, z)=\left(\mathbb{1}-{ }_{z} \mathbf{K}_{\beta}\right)^{-1} z \alpha,
$$

with $\alpha\left(x_{1}\right)=1, \alpha(x)_{n}=0$ for $n>1$.

\section{Estimates}

In this section we prove some estimates which we shall use to bound the $\beta$-derivatives of $\rho$.

Proposition 3.1. For any $\varepsilon>0$ there is a $R_{T}>0$ such that $C(\beta) \leqq \varepsilon$ for $|\beta| \leqq R_{T}$. Proof. By

$$
\left|e^{-\beta \Phi(x)}-1\right| \leqq e^{|\beta||\Phi(x)|}-1 \leqq e^{\left.|\beta||\Phi|\right|_{\infty}}|\beta||\Phi(x)|
$$


we have

$$
\int\left|e^{-\beta \Phi(x)}-1\right| d x \leqq|\beta| e^{|\beta||| \Phi \mid \|_{\infty}}\|\Phi\|_{1} .
$$

Proposition 3.2. For $R_{F}>0, d>0, \xi>\eta>R_{F}+d$, there is a $R_{T}>0$ such that for $|\beta| \leqq R_{T}, \zeta \in[\eta, \xi]$

$$
e^{-2 B \operatorname{Re} \beta} \zeta \exp [-\zeta C(\beta)] \geqq R_{F}+d
$$

Proof. By

$$
e^{-2 B \operatorname{Re} \beta} \zeta \exp [-\zeta C(\beta)] \geqq e^{-2 B \operatorname{Re} \beta} \eta \exp [-\zeta C(\beta)]
$$

this follows from Proposition 3.1.

Proposition 3.3. For $R_{F}, \xi, \eta, d, R_{T}$ as in Proposition 3.2.

$$
L=\sup _{\zeta \in[\eta, \xi]}\left\|\left(\mathbb{1}-z \mathbf{K}_{\beta}\right)^{-1}\right\|_{\zeta}^{\zeta} \leqq 1+\frac{R_{F}}{d},
$$

if $|z| \leqq R_{F}, \beta \in S_{R_{T}}=\left\{\beta|| \beta \mid \leqq R_{T}, \operatorname{Re} \beta \geqq 0\right\}, \zeta \in[\eta, \xi]$.

Proof. By Proposition 3.2. and (12)

$$
\left\|\mathbf{K}_{\beta}\right\|_{\zeta}^{\zeta} \leqq \frac{1}{R_{F}+d} .
$$

Thus the power series expansion of $\left(\mathbb{1}-{ }_{z} \mathbf{K}_{\beta}\right)^{-1}$ converges in the \|\|$_{\zeta}^{\zeta}$ - norm and

$$
\left\|\left(\mathbb{1}-z \mathbf{K}_{\beta}\right)^{-1}\right\|_{\zeta}^{\zeta} \leqq \sum_{n=0}^{\infty}\left(\frac{R_{F}}{R_{F}+d}\right)^{n}=1+\frac{R_{F}}{d} .
$$

Equation (14) yields

$$
\left\|D_{\beta}^{r} \rho(\beta, z)\right\|_{\xi} \leqq\left\|D_{\beta}^{r}\left(\mathbb{1}-z \mathbf{K}_{\beta}\right)^{-1}\right\|{ }_{\eta}^{\xi}|z|\|\alpha\|_{\eta},
$$

consequently

$$
\begin{aligned}
& \left\|D_{\beta}^{r} \rho(\beta, z)\right\|_{\xi} \leqq r ! \sum_{\substack{r_{1}, \ldots, r_{p} \geqq 1 \\
\Sigma r_{i}=r}}|z|^{p} \|\left(1-z \mathbf{K}_{\beta}\right)^{-1} \frac{D_{\beta}^{r_{1}} \mathbf{K}_{\beta}}{r_{1} !}\left(\mathbb{1}-z \mathbf{K}_{\beta}\right)^{-1} \ldots \\
& \cdot\left(\mathbb{1}-z \mathbf{K}_{\beta}\right)^{-1} \frac{D_{\beta}^{r_{p} \mathbf{K}_{\beta}}}{r_{p} !}\left(\mathbb{1}-z \mathbf{K}_{\beta}\right)^{-1} \| \eta_{\eta}^{\xi}|z| \eta^{-1} \\
& \leqq|z| \eta^{-1} L r ! \sum_{\substack{r_{1}, \ldots, r_{p} \geqq 1 \\
\sum r_{i}=r}}(|z| L)^{p} \prod_{i=1}^{p} \frac{\left\|D_{\beta}^{r_{i}} \mathbf{K}_{\beta}\right\|_{\zeta_{i-1}}^{\zeta_{i}}}{r_{i} !},
\end{aligned}
$$

where we take

$$
\zeta_{i}=\eta \cdot\left(\frac{\xi}{\eta}\right)^{(1 / r) \cdot \sum_{j=1}^{i} r_{j}}
$$

$D_{\beta}^{r} \mathbf{K}_{\beta}$ can be calculated explicitly:

$$
\left(\mathrm{D}_{\beta}^{r} \mathbf{K}_{\beta} \varphi\right)\left(x_{1}\right)=\sum_{n=1}^{\infty} \frac{1}{n !} \int d(y)_{n} D_{\beta}^{r} K_{\beta}\left(x_{1},(y)_{n}\right) \varphi(y)_{n}
$$




$$
\begin{aligned}
\left(D_{\beta}^{r} \mathbf{K}_{\beta} \varphi\right)(x)_{m}= & {\left[-W^{i}(x)_{m}\right]^{r} \exp \left[-\beta W^{i}(x)_{m}\right] \varphi(x)_{m-1}^{\prime} } \\
& +\sum_{s=0}^{r}\left(\begin{array}{l}
r \\
s
\end{array}\right)\left[-W^{i}(x)_{m}\right]^{r-s} \exp \left[-\beta W^{i}(x)_{m}\right] \\
& \cdot\left[\sum_{n=1}^{\infty} \frac{1}{n !} \int d(y)_{n} D_{\beta}^{s} K_{\beta}\left(x_{i},(y)_{n}\right) \varphi\left((x)_{m-1}^{\prime},(y)_{n}\right)\right] .
\end{aligned}
$$

Proposition 3.4. For any $R_{T}>0$ there are constants $K_{1}, K_{2}\left(R_{T}\right)$ such that for $|\beta| \leqq R_{T}$

$$
\int d(y)_{n}\left|D_{\beta}^{s} K_{\beta}\left(x,(y)_{n}\right)\right| \leqq K_{1}^{s} s ! K_{2}^{n} .
$$

Proof

$$
\begin{aligned}
& \int d(y)_{n}\left|D_{\beta}^{s} K_{\beta}\left(x,(y)_{n}\right)\right| \\
& \leqq \sum_{\substack{s_{1}, \ldots, s_{n} \geqq 0 \\
\Sigma s_{i}=s}} \frac{s !}{s_{1} ! \cdot \ldots \cdot s_{n} !} \prod_{i=1}^{n} \int d y_{i}\left|D_{\beta}^{s_{i}}\left(e^{-\beta \Phi\left(x-y_{i}\right)}-1\right)\right| \\
& \leqq \sum_{\substack{l=1 \\
\min (s, n)}}^{n}\left(\begin{array}{l}
n \\
l
\end{array}\right) C(\beta)^{n-l} . \\
& \quad \cdot \sum_{\substack{s_{1}, \ldots, s_{l} \geqq 1 \\
\Sigma s_{i}=s}} \frac{s !}{s_{1} ! \cdot \ldots \cdot s_{l} !} \prod_{i=1}^{l} \int d y|\Phi(x-y)|^{s_{i}} e^{-\operatorname{Re} \beta \Phi(x-y)} .
\end{aligned}
$$

As

$$
\int d y|\Phi(x-y)|^{s_{i}} e^{-\operatorname{Re} \beta \Phi(x-y)} \leqq\|\Phi\|_{\infty}^{s_{i}-1} e^{|\beta|\|\Phi\|_{\infty}}\|\Phi\|_{1},
$$

and

$$
\sum_{\substack{s_{1}, \ldots, s_{l} \geqq 1 \\
\Sigma s_{i}=s}} \frac{s !}{s_{1} ! \cdot \ldots \cdot s_{n} !} \leqq s ! \sum_{\substack{s_{1}, \ldots, s_{l} \geqq 1 \\
\Sigma s_{i}=s}} 1=s !\left(\begin{array}{c}
s-1 \\
l-1
\end{array}\right) \leqq s^{s} s !
$$

this leads to

$$
\begin{aligned}
& \int d(y)_{n}\left|D_{\beta}^{s} K_{\beta}\left(x,(y)_{n}\right)\right| \\
& \leqq\left(2\|\Phi\|_{\infty}\right)^{s} s ! \sum_{l=1}^{n}\left(\begin{array}{l}
n \\
l
\end{array}\right) C(\beta)^{n-l}\left(\frac{e^{|\beta|\|\Phi\|_{\infty}\|\Phi\|_{1}}}{\|\Phi\|_{\infty}}\right)^{l} \\
& \leqq\left(2\|\Phi\|_{\infty}\right)^{s} s !\left(C(\beta)+\frac{e^{|\beta|\|\Phi\|_{\infty}}\|\Phi\|_{1}}{\|\Phi\|_{\infty}}\right)^{n} \\
& \leqq\left(2\|\Phi\|_{\infty}\right)^{s} s !\left[e^{R_{T}\|\Phi\|_{\infty}}\|\Phi\|_{1}\left(R_{T}+\frac{1}{\|\Phi\|_{\infty}}\right)\right]^{n} \text {. }
\end{aligned}
$$

The last inequality follows from (16). 
Proposition 3.5. For $\beta \in S_{R_{T}}, \varphi \in E_{\zeta}$

$$
\begin{gathered}
\int d(y)_{n}\left|D_{\beta}^{s} K_{\beta}\left(x_{1},(y)_{n}\right) \varphi\left((x)_{m-1}^{\prime},(y)_{n}\right)\right| \\
\leqq\|\varphi\|_{\zeta} \zeta^{m-1} K_{1}^{s} s !\left[\zeta K_{2}\left(R_{T}\right)\right]^{n} \\
\left|\left[-W^{i}(x)_{m}\right]^{s} \exp \left[-\beta W^{i}(x)_{m}\right]\right| \leqq e^{2 B R_{T}}\left(\frac{K_{1}}{2}\right)^{s}(m-1)^{s} .
\end{gathered}
$$

Proof.

(32) is obvious from Proposition 3.4. and (5), (33) from (10).

Lemma 3.6. For $e^{-1} \xi \leqq \eta<\xi$ there is a constant $K_{3}\left(R_{T}, \eta, \xi\right)$ such that for $\beta \in S_{R_{T}}$, $\xi \geqq \zeta_{2}>\zeta_{1} \geqq \eta$

$$
\left\|D_{\beta}^{r} \mathbf{K}_{\beta}\right\|_{\zeta 1}^{\zeta 2} \leqq K_{3}\left(2 K_{1}\right)^{r}\left(\log \frac{\zeta_{2}}{\zeta_{1}}\right)^{-r} r !
$$

Proof. For $m>1$, by Proposition 3.5. and (26)

$$
\begin{aligned}
& \left|\left(D_{\beta}^{r} \mathbf{K}_{\beta} \varphi\right)(x)_{m}\right| \\
& \quad \leqq e^{2 B R_{T}}\|\varphi\|_{\zeta_{1}} \zeta_{1}^{m-1}\left[\left(\frac{K_{1}}{2}\right)^{r}(m-1)^{r}\right. \\
& \left.\quad+\sum_{s=0}^{r}\left(\begin{array}{l}
r \\
s
\end{array}\right)\left(\frac{K_{1}}{2}\right)^{r-s}(m-1)^{r-s} K_{1}^{s} s ! \cdot \sum_{n=1}^{\infty} \frac{1}{n !}\left(\zeta_{1} K_{2}\right)^{n}\right] \\
& \leqq \\
& \quad e^{2 B R_{T}+\zeta_{1} K_{2}}\|\varphi\|_{\zeta_{1}} \zeta_{1}^{m-1} K_{1}^{r}\left[(m-1)^{r}+\sum_{s=1}^{r}\left(\begin{array}{l}
r \\
s
\end{array}\right)(m-1)^{r-s} s !\right] .
\end{aligned}
$$

By

$$
\sup _{m \geqq 1} m^{s}\left(\frac{\zeta_{1}}{\zeta_{2}}\right)^{m} \leqq\left(\log \frac{\zeta_{2}}{\zeta_{1}}\right)^{-s} e^{-s} s^{s} \leqq\left(\log \frac{\zeta_{2}}{\zeta_{1}}\right)^{-s} s !
$$

we obtain

$$
\begin{gathered}
\left\|D_{\beta}^{r} \mathbf{K}_{\beta}\right\|_{\zeta_{1}}^{\zeta_{2}} \leqq \zeta_{2}^{-1} e^{2 B R_{T}+\zeta_{1} K_{2}\left(R_{T}\right)} K_{1}^{r} r ! \sum_{s=0}^{r}\left(\log \frac{\zeta_{2}}{\zeta_{1}}\right)^{-s} \\
\leqq \eta^{-1} e^{2 B R_{T}+\zeta K_{2}\left(R_{T}\right)}\left(2 K_{1}\right) r\left(\log \frac{\zeta_{2}}{\zeta_{1}}\right)^{-r} r ! \square
\end{gathered}
$$

Theorem 3.7. For $z \in \mathbb{C},|z| \leqq R_{F}, \xi>R_{F}$ there are constants $R_{T}>0, K_{4}\left(R_{T}, R_{F}, \xi\right)$ such that for $\beta \in S_{R_{T}}$

$$
\left\|D_{\beta}^{r} \rho(\beta, z)\right\|_{\xi} \leqq|z| K_{4}^{r} r !^{2} \text {. }
$$

Proof. As $\xi>R_{F}$, we can find $\eta, d, R_{T}$ as in proposition 3.2., $\eta \geqq e^{-1} \xi$. Thus, by 
(23), (24) and lemma 3.6.

$$
\begin{aligned}
& \left\|D_{\beta}^{r} \rho(\beta, z)\right\|_{\xi} \leqq|z| \eta^{-1} L\left(2 K_{1}\right)^{r} r ! \\
& \cdot \sum_{\substack{r_{1}, \ldots, r_{p} \geqq 1 \\
\Sigma r_{i}=r}}\left(R_{F} L K_{3}\right)^{p} \prod_{i=1}^{p}\left[\log \left(\frac{\xi}{\eta}\right)^{r_{i} / r}\right]^{-r_{i}} \\
& \leqq|z| \eta^{-1} L\left[2 K_{1} \max \left(1, R_{F} L K_{3}\right)\right]^{r}\left(\log \frac{\xi}{\eta}\right)^{-r} r ! \\
& \quad \sum_{\substack{r_{1}, \ldots, r_{p} \geqq 1 \\
\sum r_{i}=r}} \prod_{i=1}^{p}\left(\frac{r}{r_{i}}\right)^{r_{i}} \\
& \leqq|z| \eta^{-1} L\left[4 e K_{1} \max \left(1, R_{F} L K_{3}\right)\right]^{r}\left(\log \frac{\xi}{\eta}\right)^{-r} r !^{2},
\end{aligned}
$$

where we have used

$$
\sum_{\substack{r_{1}, \ldots, r_{p} \geqq 1 \\
\Sigma r_{i}=r}} \prod_{i=1}^{p}\left(\frac{r}{r_{i}}\right)^{r_{i}} \leqq r^{r} \sum_{\substack{r_{1}, \ldots, r_{p} \geqq 1 \\
\Sigma r_{i}=r}} 1 \leqq r^{r} \sum_{p=1}^{r}\left(\begin{array}{c}
r-1 \\
p-1
\end{array}\right) \leqq(2 e)^{r} r ! .
$$

Theorem 3.8. For $z, \beta, \xi$ as in Theorem 3.7.

$$
\left|D_{\beta}^{r}(\beta p(\beta, z))\right| \leqq|z| \xi K_{4}^{r} r !^{2}
$$

where $p(\beta, z)$ is the thermodynamic limit of the pressure.

Proof. From Theorem 3.7. it follows that $\rho_{1}(x)$, which is translation-invariant, i.e. a constant, satisfies

$$
\left|D_{\beta}^{r} \rho_{1}(\beta, z)\right| \leqq|z| \xi K_{4}^{r} r !^{2}
$$

For $|z|<e^{-2 B \operatorname{Re} \beta} \xi \exp [-\xi C(\beta)] \frac{\rho_{1}(\beta, z)}{z}$ is analytic in $z$ by (14) and

$$
\beta p(\beta, z)=\int_{0}^{z} \frac{d z^{\prime}}{z^{\prime}} \rho_{1}\left(\beta, z^{\prime}\right)
$$

(see [2], ch.4.3.). Consequently

$$
\left|D_{\beta}^{r}(\beta p(\beta, z))\right| \leqq\left|\int_{0}^{z} d z^{\prime} \xi K_{4}^{r} r !^{2}\right|=|z| \xi K_{4}^{r} r !^{2}
$$

\section{Borel-summability}

Theorem 4.1. For $z \in \mathbb{C},|z| \leqq R_{F}, \xi>R_{F}, f$ a continuous linear functional on $E_{\xi}$ there is a $R_{T}>0$ such that for $\beta$ in the circle $C_{R_{T}}=\left\{\beta \mid \operatorname{Re} \beta^{-1} \geqq R_{T}^{-1}\right\}$ the Borelsums of the Taylor-series in $\beta$ at the origin for the functions

$$
\begin{aligned}
f(\rho(\beta, z)): S_{R_{T}} & \rightarrow \mathbb{C} \\
\beta p(\beta, z): S_{R_{T}} & \rightarrow \mathbb{C}
\end{aligned}
$$

converge absolutely and uniformly in $\beta, z$.

Proof. This follows from Theorems 3.7., 3.8. and Nevanlinna's theorem ([3], see also [4]). 
Corollary 4.2. Let $R_{F}, \xi, R_{T}$ be as in Theorem 4.1., $A$ a set of finite Lebesguemeasure in $\mathbb{R}^{v n}\left(\mathrm{e}\right.$.g. a product of balls centered at points $x_{1}, \ldots, x_{n}$ ). Then the Borelsum of the Taylor-series in $\beta$ at the origin for

$$
\int_{A} \rho\left((x)_{n} ; \beta, z\right) d(x)_{n}
$$

converges absolutely and uniformly in $\beta, z$ for $\beta \in C_{R_{T}},|z| \leqq R_{F}$.

Proof. This is a direct consequence of Theorem 4.1., as the mapping

$$
\varphi \mapsto \int_{A} \varphi(x)_{n} d(x)_{n}
$$

is obviously a continuous linear functional on $E_{\xi}$.

Remark. If $\Phi$ is a continuous function, the $\rho\left((x)_{n} ; \beta, z\right)$ are continuous in $(x)_{n}$ $\left([2]\right.$, p.79) and we can replace the integral in corollary 4.2 . by $\rho\left((x)_{n} ; \beta, z\right),(x)_{n}$ fixed.

Acknowledgement. The author is much indebted to the referee for pointing out an error in the original manuscript.

\section{References}

1. Lebowitz, J. L.; Penrose, O.: Commun. Math. Phys. 11, 99-124 (1968)

2. Ruelle, D. : Statistical Mechanics. Rigorous Results. New York : Benjamin 1969

3. Nevanlinna, F.: Ann. Acad. Sci. Fenn. Ser. A12 (1918)

4. Sokal, A. : J. Math. Phys. 21, 261-263 (1980)

Communicated by E. Lieb

Received March 30, 1981 
